legten Befunde adressieren eine Vielzahl theoretischer Fragestellungen und sind von Relevanz nicht nur für die Forschung zur Internationalisierung von Kommunikation, sondern auch für die Öffentlichkeitssoziologie, die politische Kommunikationsforschung und die Europaforschung.

Allerdings sind auch einige Kritikpunkte angebracht. Ein erster, schon angedeuteter ist, dass sich kein klares, einheitliches Bild der Ergebnisse zeigt. Natürlich lässt sich nicht kritisieren, dass eine komparative Analyse der öffentlichen Kommunikation über das Mehrebenensystem EU komplexe Ergebnisse zeitigt. Aber insgesamt hätte man den Band stringenter organisieren können, und auch das abschlieBende Resümee leistet dies nicht: Die Befunde werden entlang von vier ,possible paths to a Europeanized public politics“ (Statham, 227) resümiert - und damit auf vier Modelle verteilt, die erst an dieser Stelle eingeführt werden und den empirischen Teil des Buches nicht anleiteten.

Ein weiterer kritischer Punkt ist, dass die zugrunde liegenden Daten unterausgewertet bleiben. Die komplexe "claim-making analysis“ wird nahezu durchweg bivariat ausgewertet und einzelne claim-Komponenten - etwa claim-Absender, -inhalte und -Adressaten werden kaum systematisch miteinander verbunden. Dies schmälert nicht die Qualität des präsentierten Materials, lässt aber den Eindruck entstehen, dass Möglichkeiten ungenutzt blieben.

Daneben gibt es kleinere Kritikpunkte: Ein Teil der Befunde ist, da das Projekt schon 2004 abgeschlossen wurde, bereits andernorts veröffentlicht worden und entsprechend schon bekannt. Etwas mehr Informationen zu den neben der „claim“-Analyse verwendeten Methoden wären mitunter hilfreich gewesen - so bleibt der Leser bspw. im Unklaren darüber, welche Art (qualitativer?) Analyse den „beyond numbers"-Erörterungen von Medrano und Gray (213ff.) zugrunde liegt. Schließlich wäre es wünschenswert gewesen, wenn die Autorinnen und Autoren durchgängig konsequent und klar zwischen „Europa“ und der EU unterschieden hätten.

Mike S. Schäfer

\section{Swantje Lingenberg}

\section{Europäische Publikumsöffentlichkeiten}

Ein pragmatischer Ansatz

Wiesbaden : VS, 2010. - 284 S.

ISBN 978-3-531-17166-1

(Zugl.: Erfurt, Univ., Diss., 2009)

Zur Vielzahl an Arbeiten über die europäische Öffentlichkeit gesellt sich nun ein weiterer Band. Das ist deswegen eine erfreuliche Nachricht, weil es tatsächlich gelungen ist, eine neue Perspektive auf das Thema anzulegen. Swantje Lingenberg stellt das europäische Publikum in den Mittelpunkt und überwindet damit die vorherrschende enge Fokussierung der Forschung auf medial-vermittelte Inhalte.

Der Band gliedert sich in drei Abschnitte. Lingenberg beginnt mit einer etwa 100 Seiten umfassenden Aufarbeitung des Forschungsstandes zur europäischen Öffentlichkeit. Ein kürzerer Abschnitt von etwa 50 Seiten befasst sich mit der "Theoretisierung europäischer Öffentlichkeit". Die Verfasserin knüpft dabei vor allem an John Dewey an, der das Publikum als Teil von Öffentlichkeit hervorhebt. Auf weiteren etwa 50 Seiten wird eine qualitative Befragung zur Wahrnehmung der Verfassungsdebatte durch das Publikum in Deutschland, Frankreich und Italien vorgestellt.

Die theoretischen Überlegungen setzen bei der mangelnden Berücksichtigung nicht-medialer Öffentlichkeiten im liberal-repräsentativen Modell von Öffentlichkeit an. Mit dem Diskursmodell, so Lingenberg, rückten die Zivilgesellschaft und auch nicht mediale Öffentlichkeiten zwar stärker in den Vordergrund, aber lebensweltliche Bezüge blieben unberücksichtigt. Wenn die soziale Realität angemessen erfasst werden solle, müssten aber vor allem die kommunikativen Anschlusshandlungen und die Aneignungspraktiken des Publikums im Alltag reflektiert werden (S. 228). Hier böten die cultural studies viele Anknüpfungspunkte.

Lingenberg argumentiert, dass Offentlichkeit ohne Publikum nicht denkbar ist. Auch europäische Öffentlichkeit werde nicht zuletzt durch die kommunikativen Anschlusshandlungen der Menschen hergestellt. Wie das Publikum in europapolitischen Fragen Öffentlichkeit konstituiert, untersucht Lingenberg im empirischen Teil des Bandes. Als theoretischen Rahmen stellt sie einen „pragmatischen Ansatz europäischer Öffentlichkeit" vor, den sie aus der Darstellung des Forschungsstandes zur europäischen Öffentlichkeit und aus der Auseinandersetzung mit den Überlegungen Deweys entwickelt. Er umfasst die drei Dimen- 
sionen Raumbezug, politische Zielfunktionen und Publikumsebene. Europäische Öffentlichkeit wird als Interaktionsraum beschrieben, in dem Bürger und politische Eliten Probleme verhandeln. Die Diskurse führen nicht notwendig zu einem Konsens, sondern lassen sich als Prozesse kollektiven Lernens begreifen. Europäische Öffentlichkeit liegt nach diesem Konzept dann vor, wenn das Publikum, das Bestandteil der themen- und ereignisbezogenen Teilöffentlichkeiten ist, seine Betroffenheit in europapolitischen Fragen wahrnimmt und in entsprechende Diskurse eintritt. Damit rückt Lingenberg die alltägliche Relevanz von Europa für das Publikum in den Mittelpunkt ihrer Überlegungen.

Die Ausführungen zu Dewey und die Entwicklung des dreidimensionalen Konzepts pragmatischer Öffentlichkeit sind im Verhältnis den Abschnitten zur empirischen Umsetzung sehr umfangreich geraten. Während das Konzept der Publikumsorientierung weiterführend ist und so bislang nicht reflektiert wurde, enthalten die Überlegungen zum Raumbezug sowie zu den politischen Zielfunktionen keine wirklich neuen Ideen, die den Umfang der Ausführungen rechtfertigen würden. Außerdem spielen diese beiden Dimensionen in der empirischen Studie kaum eine Rolle. Diese vergleichsweise umfassenden theoretischen Überlegungen, von denen nur ein kleiner Teil auch empirisch umgesetzt wurde, erklären sich vermutlich aus der Genese des Bandes. Es handelt sich um die gekürzte Fassung von Lingenbergs Promotionsschrift, die sie 2008 an der Universität Erfurt eingereicht hat.

Problematisch ist die ausschließlich öffentlichkeitstheoretische Fundierung, da es im Kern um die Aneignungsprozesse und die Anschlusskommunikation des Publikums geht. Die Berücksichtigung der alltäglichen Relevanz europapolitischer Fragen für das Publikum ist zwar originell und zielführend im Kontext europäischer Integration im Allgemeinen und der Herausbildung einer europäischen Identität im Besonderen. Öffentlichkeitstheoretische Konzepte sind aber wenig geeignet, um Nutzung und Rezeption in nicht-öffentlichen Kontexten zu erklären. Die Ausweitung des Öffentlichkeitsbegriffs auf die zumeist nicht-öffentliche Anschlusskommunikation hätte hier ausführlicher diskutiert werden müssen.

Die empirische Studie untersucht die Wahrnehmung und Verarbeitung der Debatte zur Europäischen Verfassung durch das Publikum. In qualitativen Interviews mit 72 Bürgern und Bürgerinnen in Deutschland, Frankreich und Italien untersucht sie die Wahrnehmung der
Betroffenheit durch die Verfassung bzw. durch die gescheiterten Referenden sowie die daraus resultierende Anschlusskommunikation, die länderübergreifende Konvergenz von Themen und Argumenten sowie die kulturellen Differenzen zwischen den drei Untersuchungsländern. Die Ergebnisse sind kaum überraschend, da sie dem meist auf Medieninhaltsanalysen beruhenden Forschungsstand zu themen- und ereigniszentrierten Teilöffentlichkeiten wenig hinzufügen. Vor allem Konflikte und Krisen bilden Anlässe für grenzüberschreitende Kommunikation, die Themen und Argumente konvergieren zwischen den untersuchten Ländern bei gleichzeitig stabilen nationalstaatlich oder kulturell geprägten Sichtweisen. Damit weicht die europäische Publikumsöffentlichkeit nicht grundsätzlich von der europäischen Medienöffentlichkeit ab. Hier fehlen leider Überlegungen zu den erwarteten Unterschieden und Gemeinsamkeiten zwischen Medien- und Publikumsöffentlichkeit. Schließlich erscheint es wenig plausibel, dass das Publikum angesichts der mangelnden Primärerfahrung ganz anderen Aspekten Relevanz zuweist als deren mediale Informationsquellen. Positiv hervorzuheben ist allerdings, dass eine mögliche empirische Umsetzung exemplarisch vorgeführt wurde und dass die Befunde durch die vielen Zitate aus den Interviews eine große Plastizität erhalten.

Insgesamt ist der Band sowohl für Einsteiger als auch für Fortgeschrittene im Bereich Europäische Öffentlichkeit ein Gewinn. Das liegt allerdings nicht so sehr an den wenig überraschenden Befunden der empirischen Studie, sondern vielmehr am ersten und zweiten Teil des Bandes. Lingenberg gibt einen gut strukturierten und umfassenden Überblick über den Stand der Forschung und bringt vor allem durch den Rekurs auf Dewey klassische Konzepte wieder auf die Tagesordnung. Besonders verdienstvoll ist die Erweiterung der Perspektive auf das Publikum.

Christiane Eilders

\section{Tilmann Sutter \\ Medienanalyse und Medienkritik}

Forschungsfelder einer konstruktivistischen Soziologie der Medien

Wiesbaden: VS, 2010. - $232 \mathrm{~S}$.

ISBN 978-3-531-16910-1

Der Autor versammelt hier 16 Aufsätze zu einer konstruktivistischen Theorie der Mediensozialisation, die die Bezüge zwischen Interaktion, Kommunikation, Massenkommunikation und 